\title{
Philosophiques
}

\section{Relativité, determinatio et parallaxe, remarques sur le traitement cartésien de trois controverses scientifiques}

\section{Vincent Jullien}

Volume 38, numéro 2, automne 2011

URI : https://id.erudit.org/iderudit/1007461ar

DOI : https://doi.org/10.7202/1007461ar

Aller au sommaire du numéro

Éditeur(s)

Société de philosophie du Québec

ISSN

0316-2923 (imprimé)

1492-1391 (numérique)

Découvrir la revue

Citer cet article

Jullien, V. (2011). Relativité, determinatio et parallaxe, remarques sur le traitement cartésien de trois controverses scientifiques. Philosophiques, 38(2), 493-521. https://doi.org/10.7202/1007461ar
Résumé de l'article

Trois sujets de philosophie naturelle, fort controversés à l'époque où Descartes élabore sa physique (incluant son système du Monde) sont particulièrement présents dans les Principes de la philosophie de Descartes, le principe de relativité des mouvements des corps matériels, la nature que l'on nommera plus tard vectorielle de la grandeur, qui caractérise l'état de mouvement d'un corps et que Descartes contribue à constituer par la notion de determinatio et l'objection parallactique contre l'héliocentrisme. On examine ici comment l'actualité du débat scientifique permet de comprendre l'argumentation cartésienne et on insiste sur le caractère polémique de ce traité. On peut notamment relever que les théories galiléennes pèsent d'un grand poids dans la modification des conceptions cartésiennes du mouvement et que les arguments, alors les plus récents, avancés en astronomie soutiennent la mise au point du système du monde cartésien. Il s'agit de mettre l'histoire des sciences au service d'une meilleure intelligence de la somme philosophique cartésienne. 


\title{
Relativité, determinatio et parallaxe, remarques sur le traitement cartésien de trois controverses scientifiques
}

\author{
VINCENT JULLIEN \\ Université de Nantes \\ Vincent-jullien@wanadoo.fr
}

\begin{abstract}
RÉSUMÉ. - Trois sujets de philosophie naturelle, fort controversés à l'époque où Descartes élabore sa physique (incluant son système du Monde) sont particulièrement présents dans les Principes de la philosophie de Descartes, le principe de relativité des mouvements des corps matériels, la nature que l'on nommera plus tard vectorielle de la grandeur, qui caractérise l'état de mouvement d'un corps et que Descartes contribue à constituer par la notion de determinatio et l'objection parallactique contre l'héliocentrisme. On examine ici comment l'actualité du débat scientifique permet de comprendre l'argumentation cartésienne et on insiste sur le caractère polémique de ce traité. On peut notamment relever que les théories galiléennes pèsent d'un grand poids dans la modification des conceptions cartésiennes du mouvement et que les arguments, alors les plus récents, avancés en astronomie soutiennent la mise au point du système du monde cartésien. II s'agit de mettre l'histoire des sciences au service d'une meilleure intelligence de la somme philosophique cartésienne.
\end{abstract}

\begin{abstract}
Three very controversial points in natural philosophy playan important role in the cartesian Principes de la philosophie in which Descartes is working his Physic (including his system of the world). There are the Relativity principle for the moving bodies, the nature of magnitude which later shall be named vectorial (named determinatio by Descartes) and the parallactic objection against heliocentric system. In this paper, it is discussed of how the scientific actuality is important for one interested in understanding the cartesian natural philosophy. It is insisted on the controversial nature of the cartesian treatise: the galilean theories have a strong influence on the modifications operated by Descartes about bodies' movement; the most performing results in astronomy in this period strenght the cartesian way to imagine a new an coherent system of the world. The main point of this paper consists in using the history of sciences in order to better understand the philosophic summa of Descartes.
\end{abstract}

MOTS-CLÉS: Descartes, Géométrie, Principes de la philosophie, Inertie, Relativité du mouvement, Parallaxe, determinatio, système du monde.

L'élaboration et l'écriture des Principia Philosophiae (puis la mise au point de la traduction française les Principes de la philosophie) s'inscrivent dans un contexte d'intense production scientifique et de multiples et profondes controverses. Le projet philosophique cartésien en est complètement imprégné et s'engage très nettement sur la plupart de ces débats. Le traité cartésien sera d'ailleurs un des textes les plus influents, les plus marquants 
de la physique du XviI et d'une bonne partie du XviII ${ }^{e}$ siècle, soit directement, soit par le biais de reprises et d'adaptations dont la plus célèbre est celle de Jacques Rohault. Son Traité de physique, publié en I67I, fut réimprimé de nombreuses fois jusque vers $\mathrm{I} 750$, il fut traduit en latin puis en anglais ${ }^{1}$.

Une caractéristique de l'intervention cartésienne sur les sujets de physique est la suivante: elle a des fondations métaphysiques stables et arrêtées alors que le traitement effectif de ces sujets, leur édification en théorie, bref l'élaboration des concepts que réclame la nouvelle science du monde visible est incertaine et paraît même inachevée ou hésitante².

Il s'agit, dans les pages qui suivent, d'examiner trois notions de grande importance pour la physique classique naissante et dont la présence est peu discutable dans les Principes même si elles ne sont pas nommées du nom qui les fera reconnaître dans l'histoire des sciences. Ces trois-là associent si fortement la philosophie première de Descartes à sa philosophie seconde qu'elles ont semblé devoir être choisies ${ }^{3}$. La première est le principe d'inertie avec le principe de relativité du mouvement (pris ensembles et on s'en expliquera); la seconde est la composition des mouvements, des vitesses et des accélérations dont la notion de determinatio est la clé cartésienne ${ }^{4}$; la troisième est la parallaxe qui constitue la plus forte objection contre le nouveau système du monde et impose que soit complètement repensée la taille du système stellaire.

Descartes s'occupe de ces sujets - en y consacrant plus ou moins de temps et de concentration - entre I629 et I647. Je mentionne les étapes bien connues de la genèse des Principes. Le I 3 novembre I 629 il déclare à Mersenne avoir pour but "d'expliquer tous les phénomènes de la nature » (I, 70). Le 22 juillet I 633, «le traité est presque achevé». On connaît bien la suite: la publication du Dialogo par Galilée suivie de la condamnation romaine conduit Descartes à ne pas rendre public son Monde 5 . Les Essais,

1. «On y voit un cartésien à l'œuvre, manipulant et travaillant les concepts du maître, introduisant des idées nouvelles et des faits nouveaux... en un mot, éclairant les Principes de Descartes par une recherche expérimentale renouvelée", Sylvain Matton, Michel Blay avec Xavier Kieft et Alain Niderst, éditeurs de Jacques Rohault, Physique nouvelle, S.É.H.A.-Archè, Milan, Paris, 2009, $4^{\text {e }}$ de couv.

2. Cela est moins vrai pour le système du monde où l'hypothèse cartésienne, telle qu'elle est développée dans $P r$. III quoiqu'il la donne pour fausse, est solidement constituée.

3. Il y aurait d'autres notions fort intéressantes à examiner sous cet angle: la théorie des trois éléments, l'aimant, la lumière, la gravité, la chaleur et la froideur, la dureté.

4. Autrement dit, il s'agit de la possibilité d'opérer (au sens de réaliser des opérations algébriques ou géométriques) sur ces grandeurs, à supposer qu'on les puisse considérer comme des grandeurs.

5. Descartes a plusieurs fois déclaré que la censure du mouvement terrestre rendait impossible la publication du Monde (à Mersenne, fin novembre I633, AT I, 27 I ; février I634, AT I, 28I-282; avril I634, AT I, 28 5-286). Voici comment il s'exprime dans la dernière de ces lettres: «Or je vous dirais que toutes les choses que j'expliquais en mon traité, entre lesquelles 
publiés en I 637 rappellent le programme général de sa physique et avancent grandement, dans la Dioptrique en particulier, sur les notions dont il est question ici. Un nouvel épisode galiléen intervient avec la publication en I638, des Discorsi que Descartes commente curieusement: de manière tout à la fois désinvolte et assez détaillée 6 . C'est dans ce contexte que Descartes entreprend la rédaction de ce qui deviendra les Principia; l'annonce en est faite à l'automne I640: "Mon dessein - écrit-il - est d'écrire toute la philosophie à partir des principes ${ }^{7}$.» Le 3 I janvier 1642, Huygens est informé que "Mon Monde se fera bientôt voir au monde [...] je veux lui faire apprendre à parler latin \& je le ferai nommer Somme philosophique ${ }^{8}$."

En I 643 et I 644 il termine la rédaction et publie son traité. Les compléments, l'Épitre dédicatoire à Elisabeth de I644, et surtout la traduction française par Picot en I 647 accompagnée de la Lettre-préface adressée au traducteur, parachèvent l'entreprise. Ces dix-huit années sont riches du point de vue de la transformation des doctrines physiques, réalisée non seulement par Descartes et Galilée, mais aussi par leurs contemporains; Gassendi, Beeckman, Cavalieri, Huygens, Roberval, Pascal, Torricelli, Harvey sont les plus connus.

\section{Le principe d'inertie et le principe de relativité}

Un argument central structure cette partie du présent article; il s'appuie sur la démonstration de l'équivalence du principe d'inertie et du principe de relativité physique (au sens restreint ou galiléen). Cette équivalence n’est, à

était aussi cette opinion du mouvement de la terre, dépendaient tellement les unes des autres, que c'est assez de savoir qu'il y en ait une qui soit fausse, pour connaître que toutes les raisons dont je me servais n'ont point de force...» (p. 285). Ainsi donc, c'est l'ensemble des explications qui est fragilisé et la cause en serait la censure romaine. Voici qui n'est pas entièrement convaincant: Descartes relève lui-même que cette censure a un statut limité et que la doctrine de Copernic continue d'avoir les faveurs de beaucoup en France et même chez des jésuites. Ne pourrait-on supposer que la fragilisation de l'ensemble de la théorie cartésienne du mouvement des corps est due, non pas d'abord à cette censure, mais au fait qu'elle a reçu, via le Dialogo des coups sévères? On aura à revenir sur la modification de cette doctrine (et des définitions du mouvement) dans les Principia. Ce serait bien un épisode galiléen qui mettrait en cause la pertinence de la publication, mais pas celui qu'on croit: ce ne serait pas la décision ecclésiale, mais plutôt l'argumentation galiléenne elle-même.

6. Pour la désinvolture, on dispose de plusieurs documents. À propos du Dialogue de I632, Descartes indique l'avoir "feuilleté » et encore en partie seulement, lorsque Beeckman le lui a prêté en août I634 (AT.I, 304). À propos des Discours, nous avons la lettre à Mersenne du 23 août I 638, dans laquelle il dit avoir «employé deux heures à le [le livre de Galilée] feuilleter et [y avoir] trouvé si peu de quoi remplir les marges etc.» (AT. II, 336). Les pages géniales de Galilée (de I 28 à I40 EN) sur l'inertie et la chute des corps, identique pour tous dans des conditions idéalisées, etc. sont expédiées par Descartes en deux lignes: "Tout ce qu'il dit icy ne peut être déterminé sans savoir ce que c'est que la pesanteur» (AT II, 385). Le traitement de toutes les démonstrations galiléennes sur le mouvement accéléré est aussi lapidaire.

7. Sept-nov-déc., I640.

8. A.T. III, 523 . 
ma connaissance, pas invoquée dans les commentateurs. Elle commande pourtant l'intelligence de la théorie cartésienne du mouvement. Il faudra donc ici justifier cette équivalence; on verra ensuite que le fond du raisonnement qui l'établit n'est pas anachronique dans les années I 640. On reviendra sur la doctrine cartésienne de l'inertie et sur les passages en rapport avec la question de la relativité physique du mouvement des corps. La tension entre la défense du principe d'inertie et ce qu'il faut bien reconnaître comme un refus de la relativité n'en sera que plus manifeste.

Voici une présentation de l'équivalence des principes d'inertie et de relativité physique des mouvements.

Le Principe d'inertie (PI) affirme qu'un corps matériel, éloigné de toute influence matérielle, n'est affecté d'aucune modification de son état de mouvement ou repos?.

Le Principe de relativité (PR) affirme qu'aucune expérience de physique ne permet de déterminer si un système matériel est au repos ou en mouvement rectiligne uniforme; soit, tous les repères en MRU sont équivalents pour décrire une expérience physique ${ }^{10}$.

I. PR implique PI.

Soit (non PI): un corps matériel ralentit dans un certain repère sans l'action d'aucune cause extérieure à lui-même. Dans ce repère, il passe de la vitesse $v$ à la vitesse $v^{\prime}$, inférieure à $v$.

Il est loisible de considérer un repère en MRU animé d'une vitesse intermédiaire entre $v$ et $v^{\prime}$. Dans ce repère, le mobile, sans action d'aucune cause, s'arrêtera et repartira en sens inverse. La nature se comporte différemment dans l'un et l'autre des repères. Les systèmes ne sont pas équivalents et PR est violé.

Donc, (non PI) implique (non PR), soit PR implique PI.

2. PI implique PR.

Soit (non PR): une cause de modification de mouvement est à l'œuvre dans un repère et ne l'est pas dans un autre repère en MRU. Autrement dit, observé dans un certain repère, un mouvement peut être modifié sans cause, ce qui est une violation de PI.

Donc, (non PR) implique (non PI), soit PI implique PR.

9. Einstein: «On sait que la loi fondamentale de la mécanique de Galilée-Newton, connue sous le nom de loi de l'inertie, est exprimée dans les termes suivants: un corps suffisamment éloigné d'autres corps persiste dans son état de repos ou de mouvement rectiligne et uniforme» (La relativité, p. 23).

10. Einstein le dira ainsi : «Les phénomènes de la nature se déroulent, relativement à K', conformément aux mêmes lois générales que relativement à K. Nous appelons cet énoncé "principe de relativité » (dans le sens restreint)» (E. 26). 
Une confirmation partielle mais suffisante (à peu de chose près) de cette relation est fournie dans les Principia de Newton. Pour celui-ci, l'équivalence des repères est un corollaire démontré (le $\mathrm{V}^{\mathrm{e}}$ ) de la loi d'Inertie (Loi I). Roger Penrose nous apprend que, dans les manuscrits newtoniens, le principe de relativité de Galilée était donné en quatrième loi de la nature ${ }^{11}$. Autrement dit, Newton s'est convaincu que PR n'était pas seulement une adjonction nécessaire à PI, mais qu'il en était une conséquence (la réciproque étant encore plus aisée à découvrir).

Il existe quelques contestations pour refuser à Descartes la paternité du principe d'inertie. Elles me semblent sans force devant les attestations textuelles aussi nombreuses que cohérentes de la thèse opposée. Descartes enseigne à ses lecteurs que, si un corps matériel est dans un certain état de mouvement (ou de repos), et n'est pas soumis à une cause extérieure à luimême, alors il conserve cet état de mouvement selon une direction droite. Dans le Monde comme dans les Principes, cela a le statut de loi de la nature (avec quelques variantes connues et bien analysées), et la thèse est répétée, plus clairement encore, dans la lettre à Huygens de février I 643:

Sur quoi je considère que la nature du mouvement est telle que lorsqu'un corps a commencé à se mouvoir, cela suffit pour faire qu'il continue toujours après avec même vitesse et en même ligne droite jusqu'à ce qu'il soit arrêté ou détourné par quelque autre cause ${ }^{12}$.

L'affaire est entendue, ce qui n'invalide pas les questions complexes qui surgissent à cette occasion: quant à l'origine métaphysique de cette loi et à son statut modal, quant à son effectivité dans un monde où, par principe, il n'existe pas de système isolé, quant à sa non-application au centre de gravité de deux corps qui se percutent, quant à la caractérisation de la ligne droite qui doit être la trajectoire du mouvement inertiel. Ces difficiles questions n'entament en rien la réalité de la présence du principe d'inertie dans la physique cartésienne. En revanche, il n'est pas possible d'y soutenir la présence du principe de relativité du mouvement, et je voudrais montrer que l'absence de ce dernier jette le trouble sur la présence du premier.

Les articles de PR II et Pr III orientent — ou désorientent - le lecteur dans deux directions qui, toutes deux, ont ceci de remarquable qu'elles nient le principe de relativité physique. La chose est soigneusement élaborée par Descartes: les deux définitions du lieu, le lieu intérieur et le lieu extérieur (II, I 3 et II, I 5) appellent et préparent les deux définitions du mouvement, selon l'usage et à proprement parler (II, 24 et II, 25). S'il s'agit du lieu extérieur, la porte est ouverte à la relativité la plus débridée: on peut décider

11. Roger Penrose, The Road to Reality. A Complete Guide to the Laws of the Universe, Jonathan Cape, 2004. Traduction française par Céline Laroche, À la découverte des lois de l'univers, O. Jacob, 2007, p.I7.

12. AT. III, 623. 
arbitrairement du lieu extérieur d'un corps comme on peut décider arbitrairement qu'il est ou non au repos. Dans le cas du lieu intérieur, la porte est complètement refermée: un corps a un lieu intérieur et un seul qui, au fond, est son attribut principal. Il est donc, soit en mouvement s'il change de voisinage, soit au repos s'il n'en change point. On constate ainsi qu'il y a là deux façons de récuser le principe de relativité physique.

Les commentateurs, et même les plus avisés, se sont parfois emmêlés dans cet écheveau, prêtant à Descartes un relativisme radical, ou alors lui déniant toute appréhension de la relativité. D’une façon générale, ils n'explicitent pas la distinction essentielle entre la relativité géométrique et la relativité physique ou Principe de relativité13.

13. F. Alquié note à propos de II.r 3 : «la négation de l'espace cadre conduit Descartes à l'idée d'une relativité absolue des mouvements» (note I, I 58), formulation, au mieux maladroite sinon fausse. Dans une note sur II.29, il affirme que: "Pour Descartes géomètre, la relativité du mouvement est donc totale» (note I, I73); mais qu'en est-il pour le Descartes physicien auquel nous avons affaire dans les Principes? Puis, commentant II. 44, il écrit: "Autrement dit, lorsque deux mouvements sont de vitesse différente, le moins rapide est comme au repos par rapport à l'autre» (n. I ; p. I95), ce qui, dit comme cela, est un emprunt direct au Principe de relativité.

A. Koyré lui-même donne des signes d'hésitation en écrivant, à deux pages d'intervalle: «Les Principes essayent de nous donner une définition physique [du mouvement], fondée sur le principe de relativité du mouvement» (p.337), puis «Descartes, pour lui-même, n'a jamais pris au sérieux et n'a jamais employé comme base de ses déductions la notion relativiste qu'il vient d'exposer si longuement. En effet ce n'est pas seulement avec les lois du choc que la relativité cinétique du mouvement se révèle incompatible. Elle l'est déjà avec celle de la conservation du mouvement, comprise, comme Descartes veut expressément la comprendre, comme conservation de la quantité de mouvement [...]» (Études galiléennes, p. 339).

Paul Mouy a bien raison de dire, en étudiant les règles du choc, que «la manière de considérer le mouvement y est en désaccord complet avec la relativité que Descartes lui avait attribuée en principe" (P. Mouy, Le développement de la physique cartésienne, p. 22); mais il n'en dit pas beaucoup plus au sujet de cette relativité de principe.

D. Garber conclut plus fermement en notant que «Il importait à Descartes de faire du mouvement un mode authentique du corps, un mode de la substance étendue [...] or si le mouvement est un mode authentique, le point de vue qui établit si un corps est en mouvement ou non ne peut être arbitraire; il doit y avoir une différence authentique entre mouvement et repos» (p. 33I). Cela est, bien entendu, un rejet absolu du principe de relativité; mais alors quid des passages physiques où le mouvement et le repos peuvent être équivalents?

Enfin (et pour en rester à la sélection faite ici), F. de Buzon et V. Carraud commentent II.24 (p. 8I) en soutenant un rapprochement plutôt audacieux: "Cela permet à Descartes d'affirmer un principe de relativité du mouvement fondé sur un exemple et un argument proches de ceux employés par Galilée, celui de la perceptibilité ou de l'imperceptibilité du mouvement d'un bateau, selon que l'observateur placé à l'intérieur considère le système avec lequel il fait corps ou l'extérieur." C'est en effet assez audacieux de soutenir une telle proximité. Justement Galilée montre l'imperceptibilité du mouvement, non seulement pour l'observateur qui regarde à l'intérieur ou à l'extérieur, mais pour tout phénomène et toute expérimentation physique; et encore faut-il prendre en considération que cette imperceptibilité ne vaut que si le mouvement est inertiel. 
L'opinion d'Alan Gabbey est plus précise à ce sujet:

A relativity principle is often attributed to Descartes. Such an attribution is misleading, if it implies a principle that mirrored the Galilean or inspired the Huygenian relativity principle ${ }^{14}$.

Le sujet qui fait problème n'est pas, en effet, la relativité cinématique ou encore géométrique. Ce n'est pas que la chose soit sans intérêt, mais elle est sans intérêt physique car elle n'a pas d'enjeu concernant la théorie du mouvement des corps matériels (personne ne songe à introduire ici des considérations qui conduiront à la théorie de la Relativité générale $)^{15}$. Descartes a rejeté de la façon la plus claire la thèse d'un repère absolu (II.I3), thèse des tenants de la voûte cosmique (les aristotéliciens comme les coperniciens stricts), mais aussi une thèse newtonienne à laquelle le savant anglais donnera une forme complètement nouvelle. On peut certes toujours décider arbitrairement une origine des mesures topologiques et des axes; on aura une géométrie plus ou moins complexe, mais la question n'est pas là; la question est évidemment celle-ci: y a-t-il des conséquences physiques au choix du repère?

Descartes reconnaît que telle est bien la question lorsque, dans l'article 24, plutôt complexe: il convoque son homme assis sur le pont d'un navire: "nous pouvons dire qu'en même temps elle [la chose et en l'occurrence l'homme assis] se meut et ne se meut point» (Alq. I69), ouvrant ainsi la voie à un relativisme du mouvement. Immédiatement, un autre point de vue est avancé:

Toutefois, à cause que sommes accoutumés de penser qu'il n'y a point de mouvement sans action, nous dirons que celui qui est ainsi assis est au repos, puisqu'il ne sent point d'action en soi, et que cela est en usage. (p. I69)

Autrement dit, si je ne sens point d'action alors je suis au repos et donc si je suis en mouvement, l'action se manifeste. À quoi peut bien songer Descartes? Si je suis sur un tourniquet rapide en rotation, je "sens effectivement» le mouvement; si je suis en chute libre, de même. Il semblerait donc qu'il y ait là un "vrai» critère du mouvement ou du repos. Mais ce critère ne fonctionne pas toujours - il manque de généralité — comme en atteste l'exemple de l'homme sur son bateau. Descartes ne peut ignorer qu'une cer-

14. Alan Gabbey, "New doctrines of motion", in The Cambridge History of Seventeenth-Century Philosophy, D. Garber and M. Ayers, ed., CUP, I998, p. 666.

15. Quoique je ne résiste pas au plaisir de reproduire l'argument formidable de M. A. Tonnelat attribuant à Descartes une certaine conception de l'espace et des corps qui s'y trouvent, telle qu'elle offre un cadre où est possible la relativité générale: «D’après la Relativité générale $[\ldots]$ les lois des phénomènes de gravitation trouvent l'interprétation la plus simple et en même temps la plus cartésienne que l'on pouvait imaginer: la réduction à l'étendue et au mouvement. " Introduction à Les principes de la théorie électromagnétique et de la relativité, Masson I959, p. 7. 
taine classe de mouvements échappe à ce critère, celle des mouvements qui sont rectilignes uniformes (MRU). Lui faudrait-il alors admettre que des choses en MRU sont au repos les unes par rapport aux autres, ce qui ne convient à aucune des définitions cartésiennes du mouvement (ni la première ni la seconde)? Il est pourtant vrai que l'indifférence des sensations et plus généralement l'indifférence des phénomènes n'est pas un critère suffisant de repos ou de mouvement. Cette indifférence est seulement un critère d'équivalence pour une classe de repères, ceux qui sont en mouvement rectiligne uniforme. Il y a des cas où le mouvement est associé à une action, une cause qui est une force extérieure au mobile, mais il y a des cas - ceux qu'on nommera inertiels - où le mouvement (ou le repos) sont sans cause actuelle. Ainsi, il est faux de dire «tous les repères sont équivalents ", mais il est faux aussi de dire «à un certain état cinématique d'un corps correspond un repère privilégié » ${ }^{16}$; il est faux de dire « une cause n'est pas nécessaire pour qu'il y ait mouvement» si celui-ci est accéléré, mais il est faux aussi de soutenir "une cause est nécessaire pour qu'il y ait mouvement », si celui-ci est inertiel.

Or cette distinction, Descartes ne la fait pas: son marin en fait trop ou en fait trop peu: l'absence de sensation ne teste pas "certains mouvements ", ceux en MRU, mais en teste certains autres, ceux qui sont accélérés. On a là une trace du principe de relativité physique, mais une trace seulement.

Comment cette remarquable découverte peut-elle avoir échappé à Descartes? On le sait (cf. n. supra), il a consulté le Dialogue sur les deux principaux systèmes du Monde et, même s'il prétend n'avoir fait que le « feuilleter » partiellement, le commentaire qu'il en fait est instructif, puisque, dit-il à Mersenne: "Je trouve qu'il [Galilée] philosophe assez bien du mouvement, encore qu'il n'y ait que fort peu de chose qu'il en dit, que je trouve entièrement véritable ${ }^{17}$.» Dans son survol, une page lui aurait-elle échappé ${ }^{18}$ ? celle, historique, où est magistralement exposé le Principe de relativité:

Enfermez-vous avec un ami dans la plus grande cabine, sous le pont d'un grand navire et prenez avec vous des mouches, des papillons et d'autres petites bêtes qui volent; munissez-vous aussi d'un grand récipient rempli d'eau avec de petits poissons; accrochez aussi un petit seau dont l'eau coule goutte à goutte dans un autre vase à petite ouverture placé en dessous. Quand le navire est immobile observez soigneusement comme les petites bêtes qui volent vont à la même vitesse dans toutes les directions de la cabine, on voit les poissons nager indifféremment de tous côtés, les gouttes qui tombent entrent toutes dans le vase placé en dessous; si vous lancez quelque chose à votre ami, vous n'aurez pas besoin de jeter plus fort dans une direction que dans l'autre quand

16. C'est toute une classe de repères qui conviennent pour y déployer la physique, les mêmes lois.

17. AT. I, 304 .

18. A. Gabbey note ainsi: "Descartes would have noticed phenomena similar to those described in the second day of the Dialogo, but he seems not to have realized their significance...", op. cit., p. 666. 
les distances sont égales, si vous sautez à pieds joints comme on dit, vous franchirez des espaces égaux dans toutes les directions. Quand vous aurez soigneusement observé cela [...] faites aller le navire à la vitesse que vous voulez; pourvu que le mouvement soit uniforme, sans balancement dans un sens ou l'autre, vous ne remarquerez pas le moindre changement dans tous les effets qu'on vient d'indiquer; aucun ne vous permettra de vous rendre compte si le navire est en marche ou immobile [...]. Si tous ces effets se correspondent, cela vient de ce que le mouvement du navire est commun à tout ce qu'il contient aussi bien qu'à l'air». (E. N. 213, op. cit. p. 204-205)

La thèse de la relativité est déjà chez Giordano Bruno, dans son «Banquet des cendres » (publié à Londres en I 584), avec même un navire ${ }^{19}$, et elle est aussi dans les Thèses jésuites de I 624 inspirées par Grégoire de SaintVincent et soutenues par deux de ses élèves ${ }^{20}$. Et on la trouve encore dans l'exposé que Mersenne fait des thèses de Galilée ${ }^{21}$. Il semble bien qu'elle emporte l'adhésion des lecteurs compétents et libérés des doctrines de l'école.

Cette théorie en voie de constitution pourrait bien être, davantage que les raisons souvent invoquées (Rome et l'immobilité de la Terre), la raison de l'hésitation cartésienne. Alexandre Koyré, comme d'autres avant lui et depuis, ont insisté sur l'argument selon lequel la relativité du mouvement est apparemment maintenue par Descartes pour «concilier l'astronomie copernicienne... avec la doctrine de l'Église ${ }^{22} »$. Je ne crois pas à son importance pour ce qui concerne la science cartésienne; lui en accorder trop serait prendre les adversaires pour des sots ou des aveugles. Il est par exemple net que l'objection biblique la plus souvent citée, concernant l'invocation de Josué, Soleil, arrête-toi au-dessus de Gabaon! relève de la relativité cinématique et pas du tout physique. On observera que les formules cartésiennes sur la terre «qui se repose en son ciel» (voir seconde partie de cet article) n'offrent pas la moindre parade à cette objection biblique contre le «mouvement de la terre »; elles ne conviennent pas à l'exploit de Josué.

On peut imaginer qu'il y voit un risque, celui de ne pas pouvoir dissocier suffisamment l'action du mouvement. En effet, l'action est radicalement le critère des mouvements non inertiels, mais pas l'action au sens des qualités de l'école, l'action en un sens nouveau, celui de la force ou de l'accélération, concept encore mal abouti au temps où Descartes conçoit sa physique. C'est ainsi que les deux définitions du mouvement ont pour point commun de ne pas enregistrer la théorie galiléenne. L'article II.24 feignait de pouvoir prendre n'importe quel repère pour définir le mouvement d'un corps, ce qui

19. Jean Dhombres, Patricia Radelet de Grave, Une mécanique donnée à voir, Brepols, 2009 , p. 20.

20. Id. La manière dont Grégoire est "une sorte de galiléen" est extraordinairement exposée, expliquée et mise en scène par les auteurs de cet ouvrage exceptionnel.

21. Les nouvelles pensées de Galilée, Paris, I639, rééd. Costabel et Lerner, Paris, Vrin, I973.

22. A. Koyré, Études galiléennes, Paris, Hermann, I966, p. 339. 
ruinait l'équivalence des repères en MRU; l'article II.25 prend le parti opposé, à savoir le choix d'un repère privilégié, associé à chaque corps, pour juger de son état de mouvement ou de repos. C'est une autre façon de ruiner ou de ne pas exploiter cette équivalence.

Si le mouvement est accéléré (comme quand on se met à courir) ou s'il combat la gravité (quand notre bras se lève), ou s'il s'oppose à la résistance de l'air, ou s'il tourne... alors, il y a bien une cause, une action, une force qu'il faut vaincre pour modifier l'état du mobile. En revanche, s'il s'agit d'un mouvement rectiligne uniforme, une telle action, cause, force est un préjugé et n'existe pas. Alors il est équivalent - du point de vue de l'action, ou de la force - de considérer le repos ou le mouvement.

L'ambiguité de la position cartésienne est visible en (II.29) «Lorsque nous verrons que deux corps qui se touchent immédiatement seront transportés, l'un d'un côté et l'autre d'un autre, et seront séparés, nous ne ferons point difficulté de dire qu'il y a tout autant de mouvement en l'un comme en l'autre» (Alq. I73). Il y a une imprécision sur la façon dont s'éloignent les deux corps: est-ce selon un mouvement rectiligne uniforme ou non?

Une remarque de Descartes revient sur cette idée:

Il n'y a rien d'absolu dans le mouvement que la séparation de deux corps en mouvement l'un avec l'autre, mais que l'on dise de l'un des corps qu'il est en mouvement, de l'autre qu'il est en repos, ceci n'est que relatif et dépend de notre manière de concevoir, comme c'est bien le cas de ce mouvement qui est appelé local: ainsi quand je marche sur la terre, tout ce qu'il y a d'absolu et de réel et de positif dans ce mouvement consiste dans la séparation de la surface de mes pieds d'avec la surface de la terre. (Remarques que M. Descartes semble avoir écrites sur les «Principes de la philosophie », trad. Costabel et Testard, AT IX-2362)

Il est juste de souligner qu'un dispositif cartésien, exposé en (II.30), permet de se sortir de l'arbitraire de la décision, de la considération que nous faisons: le critère est celui d'être mû en entier, dans sa totalité. Cet argument a toutefois sa limite: la contradiction (la Terre va à la fois vers l'Orient et vers l'Occident) n'est atteinte que si l'on considère comme absolu, réel et positif que $\mathrm{AB}$ et $\mathrm{BC}$ sont animés de mouvements contraires, ce qui n'a rien de nécessaire. Je peux considérer que $\mathrm{AB}$ est bien au repos, la conséquence en sera seulement que BC va plus vite vers l'Occident. De toute façon, cet argument ne fait pas la distinction des situations pourtant distinctes: les corps en question sont-ils en MRU ou non ${ }^{23}$ ?

23. Une fois encore les commentateurs font parfois l'impasse sur la nature des mouvements: «Pour Descartes géomètre, la relativité du mouvement est donc totale » note F. Alquié, (Alquié, note I, I73). E. Mehl, «les propositions décrivant l'événement [...] renvoient à une même cause ou force» (p. 87) puisque justement dans le mouvement (réciproque) en MRU il n'y a ni cause ni force à l'œuvre. On notera toutefois chez E. Mehl le souci de ne pas négliger les aspects scientifiques des textes cartésiens et le recours à des informations en général solides pour en traiter. 
Voyons comment ces remarques peuvent contribuer à notre lecture des règles du choc.

Les deux corps sont susceptibles de s'arrêter, de se mettre en mouvement et de changer de direction; ils ne sont donc pas en MRU durant tout l'épisode de la percussion; on ne cherchera donc pas à trouver une équivalence générale en prenant l'un des corps, puis l'autre comme repère. Il y a cependant des cas où la considération du Principe de relativité serait pertinente pour examiner la situation ou en comparer deux: lorsqu'un des corps ne change pas d'état de mouvement (ou de repos) avant ou après le choc, il est possible de le prendre pour repère, ou pas: de corps au repos, il peut "devenir» corps en mouvement et réciproquement. Selon le principe de relativité, les phénomènes ne devraient pas s'en trouver modifiés. Telle est la situation prévue par la troisième loi à l'article 40 lorsqu'un corps "plus gros" n'est pas perturbé par la percussion d'un plus petit. Considérant la situation $\mathrm{n}^{\circ} 5$ de l'article 50 , il sera possible de se rapporter à la situation de cette troisième loi (art. 40) en prenant un repère en MRU de vitesse égale à celle de B. Les conséquences cartésiennes sont pourtant annoncées comme différentes: dans un cas (cas $\mathrm{n}^{\circ} 5$, art. 50) les deux corps "repartent ensemble»; dans un autre (équivalent selon le Principe de relativité et conformément à l'article 40), ils s'écartent l'un de l'autre. C'est à nouveau le cas pour les situations $n^{\circ} 2$ et $7 \mathrm{~b}$ et encore 4 et $7 \mathrm{a}$ respectivement. Voyons par exemple les situations 4 et $7 \mathrm{a}$.

Le cas 7 a est décrit dans un certain repère $R$. Décrivons-le dans un repère $R^{\prime}$ animé d'une vitesse $b$ égale à celle qu'avait le corps $\mathrm{B}$. On se retrouve dans la situation du cas $n^{\circ} 4$ avec la même différence qualitative des effets. Les résultats cartésiens contreviennent au "principe de relativité ", disons de Galilée.

Une remarque complémentaire est nécessaire à propos de la «force de repos". La conception plus conforme à la définition "selon l'usage» des Principes était celle du Monde, comme on l'a vu. Il y avait, note A. Koyré une identification plus forte du "statut ontologique du mouvement et du $\operatorname{repos}^{24}$ ", et il s'agit, en effet, de produire une distinction entre le mouvement et le repos, si l'on veut prendre au sérieux la seconde définition du mouvement. La très délicate fin de (II.43) au sujet de la force de repos peut être interprétée notamment à l'aide du principe de relativité: on savait qu'un corps qui va à la rencontre d'un autre a une force de mouvement mesurée notamment par sa vitesse. S'il est au repos, ce ne peut plus être un paramètre valable, et c'est la vitesse de celui ou de ceux qui viennent à sa rencontre qui contribue à mesurer sa force de repos. Autrement dit, on obtient cette force, de mouvement ou de repos, en changeant de point de vue. L'article 44 dit — dans une certaine confusion - des choses du même genre. C'est ce qu'exprime clairement et en toute simplicité F. Alquié: "Autrement dit, lorsque 
deux mouvements sont de vitesse différente, le moins rapide est comme au repos par rapport à l'autre ${ }^{25}$." La "force de repos » ou encore la "tardivité qui participe de la nature du repos » (art. 44) et se mesure selon le mouvement de l'autre corps évoque irrésistiblement le principe d'équivalence des repères d'inertie. Il y a sans doute là une incursion cartésienne en terre relativiste physique.

Ces remarques convergent vers un diagnostic général assez net: quand le principe de relativité galiléenne est présent dans les Principes, c'est sous forme allusive ou alors pour être contredit.

En vertu de notre argument principal (l'équivalence des deux principes), l'affirmation cartésienne solide et assez cohérente du principe d'inertie constitue une très sérieuse objection à clore ainsi la discussion sur le traitement du principe de relativité dans ses Principes. Les contradictions que nous avons rencontrées en voulant tester la relativité du mouvement étaient inévitables puisque Descartes promeut le premier et ne soutient pas le second. Il faut préciser encore quelques aspects originaux et décisifs de l'inertie chez Descartes.

Comme on le sait, une forme non aboutie du principe d'inertie existe chez Galilée: les corps continuent à se mouvoir horizontalement si nulle cause supplémentaire n'intervient; c'est précisément cet «horizontalement" qui donne à l'inertie galiléenne son caractère circulaire, circumterrestre. Beeckman lui aussi défendait la conservation du mouvement circulaire $^{26}$. Alexandre Koyré a donc raison de dire à propos de la thèse cartésienne que: "Jamais encore le privilège du mouvement circulaire n'avait été aussi résolument et aussi simplement nié27.»

Pour comprendre cet accomplissement cartésien, il est sans doute pertinent de ne pas oublier que Descartes est, en son temps, le meilleur théoricien de la tangente à une courbe ${ }^{28}$. Il sait qu'à un point donné de trajectoire (de mouvement) d'un mobile, correspond une tangente unique et une infinité de cercles dont celle-ci est tangente. Donc, la «nécessité » selon laquelle, en chaque instant, Dieu maintient le corps en son état ne peut être exprimée par autre chose que par la tangente. Il faudrait, sinon, qu'Il calcule et choisisse en chaque instant une certaine courbure, en fonction de raisons contingentes (qui inclineraient sans nécessité, dira Leibniz). Cet aspect essentiel de l'article (II.39), plus particulièrement développé dans l'analyse de la fronde se comprend donc par la Géométrie: "le mouvement ne se fait pas en un instant, néanmoins il est évident que tout corps qui se meut [...] est déterminé à se mouvoir $[\ldots]$ suivant une ligne droite, et non pas suivant une circulaire ${ }^{29}$ ».

25. Alquié, n. I; p. 195.

26. Cf. Koyré, Études galiléennes p. 327.

27. Koyré, Études galiléennes, p. 3 I 8-34I.

28. Cf. La méthode des normales de la Géométrie, AT.VI, p. 4I3 sq.

29. Pr. II, 39, A.T. IX 2, p. 86. 
Le même argument était déjà dans le Monde ${ }^{30}$. À la question, comment concilier "qu'il n'y a pas de mouvement en un instant et que Dieu le conserve comme il est dans l'instant " ? la géométrie répond clairement. Soit un point $\mathrm{M}$ d'une trajectoire, il y a "en lui » ses coordonnées qui disent «où il est dans le mouvement "; observons que ces mêmes coordonnées ne disent rien de ce qu'il était auparavant et de ce qu'il sera par après, ainsi il n'y "pas de mouvement en un instant ». Toutefois, il y a davantage en ce point $\mathrm{M}$ que ses seules coordonnées; on y rencontre les caractéristiques de sa tangente (ou de sa normale). La résolution de ce problème est «le plus utile et le plus général, non seulement de ce que je sache, mais même que j’ai jamais désiré de savoir en Géométrie» dit Descartes en $1637^{31}$. La solution de la Géométrie de 1637 sera transformée et généralisée par le calcul différentiel; elle n'en demeure pas moins un concept cartésien: en ce point particulier M, il y a donc, isolée, sa situation, mais aussi la suite de sa trajectoire (sa tendance, car il peut être soumis à d'autres causes) ainsi que l'héritage de ce qu'il y avait avant, de ce qui l'a conduit là, en $\mathrm{M}$.

Pour revenir au principe d'inertie en général, il faut bien reconnaître qu'il est toutefois mis à mal dans les Principes puisque Descartes ne l'applique pas au centre de gravité d'un système constitué par deux corps qui se rencontrent. Une belle découverte sera celle que fera Huygens pour appliquer le Principe d'inertie à l'étude de la percussion: quels que soient les chocs et les rencontres, le centre de gravité G des corps est en MRU. Il sera donc loisible de prendre G comme origine du repère ou l' "espace environnant ». Le système pourra alors être considéré comme au repos ou non.

Si $\mathrm{v}_{\mathrm{a}}$ et $\mathrm{v}_{\mathrm{b}}$ sont les vitesses de $\mathrm{A}$ et de $\mathrm{B}$, et $\mathrm{v}_{\mathrm{g}}$ celle de centre de gravité, alors, à chaque instant, $\mathrm{V}_{\mathrm{g}}=\left(\mathrm{A} \cdot \mathrm{v}_{\mathrm{a}}+\mathrm{B} \cdot \mathrm{v}_{\mathrm{b}}\right) /(\mathrm{A}+\mathrm{B})$.

$\mathrm{V}_{\mathrm{g}}$ est donc constante si et seulement si $\left(\mathrm{A} \cdot \mathrm{v}_{\mathrm{a}}+\mathrm{B} \cdot \mathrm{v}_{\mathrm{b}}\right)$ est constante. Bref, $\mathrm{G}$ est inertiel si et seulement si la quantité de mouvement (vectorielle ou plutôt algébrique) est conservée. On distingue facilement dans les règles du choc entre celles qui respectent l'inertie du centre de gravité ( $\mathrm{I}, 5$ et $7 \mathrm{a}$ ) et les autres qui ne le respectent pas.

L'étude du mouvement des corps, et celle des rencontres selon des mouvements droits uniformes, a été consignée par Huygens dans le De motu corporum ex percussione (rédigé en 1656) ${ }^{32}$ et dans le De motu corporum ex mutuo impulsu Hypothesis de I669 ${ }^{33}$. Le principe de relativité (dit «du bateau ») y est explicite. Fabien Chareix, dans son étude désormais classique de la physique hugonienne, soutient donc que "Huygens peut et veut fonder toute la mécanique à partir de la relativité elle-même $[\ldots]^{34} »$. On notera que

30. A.T. XI, p. 46.

31. La Géométrie, Livre II, A.T. VI, 4I3.

32. Publié en I703, O. C. XVI, p. 30-9I.

33. O. C. VI, p. 336-343.

34. Fabien Chareix, La philosophie naturelle de Christian Huygens, Paris, Vrin, Mathesis, 2006. Toute la partie consacrée au «mouvement droit» expose et analyse cet acquis hugonien. 
l'hypothèse de l'inertie et celle des vitesses relatives (complétée par celle des deux observateurs dans le second texte) ne sont pas explicitement données comme équivalentes.

Si mes arguments sont valables, si PI et PR sont équivalents - et il est donc illogique de trouver l'un sans l'autre chez Descartes - , ou encore, la présence de l'un (le premier) sans l'autre ne peut manquer de soulever des contradictions. Raisonner ainsi permet de tirer une leçon essentielle du texte cartésien: quand Descartes travaille à ses Principes, lorsqu'il élabore sa physique nouvelle, ces principes et concepts sont en cours d'élaboration, leur statut profond n'est pas établi et moins encore stabilisé ou formalisé. C'est ainsi qu'il convient d'observer les efforts cartésiens pour contrer cette équivalence entre repos et MRU, pour contrer le principe de relativité que Galilée a si nettement exprimé. Ces efforts consistent à forger un critère pour dire "en propre", ce que c'est que le mouvement: c'est le "changement de voisinage ». Si un piquet est dans un courant d'eau, pourquoi est-ce lui qui est au repos et pas l'eau? Parce qu'ainsi, il y a «moins » de matière à mouvoir. Serait-ce un principe d'économie de mouvement qui déciderait de «ce qui est en mouvement" et de "ce qui est au repos». Selon l'inertie ou la relativité galiléenne, il n'y a aucun critère objectif pour en décider; la liberté est totale.

On a souvent observé l'évolution de la doctrine du mouvement entre le Monde ${ }^{35}$ et les Principia. Dans le traité de I633, la définition était quasi seulement celle de l'article 24; ainsi notent F. de Buzon et V. Carraud:

La définition selon l'usage commun est pour une part importante la définition de Descartes même dans le Monde ( Je n'en connais aucun autre que celui $[\ldots]$ qui fait que les corps passent d'un lieu à un autre»). La deuxième définition peut apparaitre [aussi comme la rectification] de l'opinion de Descartes telle qu'elle s'exprime dans l'ouvrage de $1633^{36}$.

Mais, a-t-on bien considéré que, justement, entre la date de rédaction du Monde et des Principia, il y a l'élaboration galiléenne du principe de relativité des repères en MRU?

\section{La determinatio, composition des mouvements, des vitesses et des accélérations}

La notion de determinatio qui joue un rôle important dans le traité, (dixhuit occurrences en $P r$. II) est, pour tous les traducteurs, un véritable cassetête. En français, ils ont généralement opté pour le terme le plus neutre possible, détermination qui n'a aucune correspondance avec quelque concept de la physique classique que ce soit. Le lecteur moderne a donc bien

35. Le monde, chap. VII, AT XI, 39-40, Alq. 352-353.

36. Buzon-Carraud, (p. 80-8I) citent et commentent le Monde (AT XI, 39-40; Alq. I, 352-353). 
du mal à saisir de quoi il peut être question. On verra que la notion dont on aperçoit, selon moi, la gestation dans l'usage cartésien de la determinatio, à savoir la grandeur vectorielle, commence à opérer avant que le nom de la chose qu'elle annonce ne soit forgé; elle apparaît aussi dans les expressions nombreuses où il est question de corps déterminés à se mouvoir.

Les questions soulevées par la determinatio cartésienne sont intimement liées avec certains aspects du problème précédent (le mouvement et sa propriété inertielle); quand advient cette determinatio, il s'agit en effet toujours de décrire une chose qui est à la fois ponctuelle (du point de vue de la situation) mais qui est aussi bien plus que ponctuelle, une chose caractérisée par une intensité (une grandeur positive) et une direction.

L'expression la plus nette de cette nature complexe est donnée dans $L e$ monde, lorsque Descartes y présente le modèle de la fronde:

Comme par exemple, si une pierre se meut dans une fronde, suivant un cercle marqué $\mathrm{AB}$, et que vous la considériez précisément telle qu'elle est à l'instant qu'elle arrive au point $\mathrm{A}$, vous trouvez bien qu'elle est en action pour se mouvoir, car elle ne s'y arrête pas, et pour se mouvoir vers un certain côté, car c'est là que son action est déterminée en cet instant ${ }^{37}$.

Or une telle chose - qui mesurerait "comment est la pierre à cet instant» - n'existe pas au temps où écrit Descartes, ni en géométrie ni en physique. Elle est toutefois indispensable pour décrire et comprendre les vitesses, les forces, les accélérations; il s'agit bien de la grandeur vectorielle. La determinatio cartésienne n'est pas équivalente à la grandeur vectorielle (les multiples difficultés et obscurités qui lui sont associées s'en trouveraient singulièrement simplifiées) mais elle contribue à son invention; il s'agit ici de repérer comment le concept de grandeur vectorielle est approché, d'enregistrer l'étape de sa genèse qui est franchie.

La determinatio a d'abord à voir avec la composition (et la décomposition) des mouvements, des trajectoires. C'est pourquoi on doit avoir à l'esprit les articles II.3 I et II.32 pour en saisir la nature. II.3 I est là pour frapper les esprits à partir d'un exemple commun: le mouvement des aiguilles de la montre du marinier, auxquels participent maints autres mouvements (il va et vient sur le pont, le bateau vogue, etc.) qui, en quelque sorte, le constitue. II.32 est strictement géométrique et rappelle la génération, par mouvements composés, de la cycloïde ${ }^{38}$, mais aussi - et c'est peutêtre plus intéressant - Descartes montre qu'un mouvement rectiligne peut bien être composé de deux (et donc de plusieurs) autres mouvements (droits ou non d'ailleurs) $)^{39}$.

37. A.T. XI, 45-46.

38. Sur la cycloïde...

39. On pourra rappeler le cas du trajet droit, composé de deux mouvements circulaires. On peut aussi évoquer la quadratrice d'Hippias. 
À un second niveau, il faut considérer que ces trajectoires (composées), dès qu'elles deviennent physiques, exigent que le corps qu'elles concernent aient une vitesse. La conception cartésienne de vitesse a évolué, depuis la notion préclassique de force du mouvement accompli (dans les années I 620) jusqu'à celle de vitesse ponctuelle, ou degré de vitesse, bref d'une sorte d'avatar de ce que nous nommons la vitesse instantanée ${ }^{40}$. C'est important puisque la vitesse préclassique ne laisse pas de place à la notion de determinatio.

Il est assez raisonnable d'employer pour cet objet physique (la vitesse ponctuelle) le terme cartésien somme toute assez stable de détermination à se mouvoir. Quoi qu'il en soit, en I643, la vitesse est, pour Descartes, une sorte de quantité, une caractéristique en un point de la trajectoire, ou à un instant donné du déplacement. Que les vitesses, ainsi comprises, se composent et se décomposent selon des règles analogues aux règles de décomposition du mouvement n'allait pas de soi : c'est une chose de dire que la roue du carrosse tourne et aussi avance, engendrant une cycloïde, c'en est une autre que d'analyser la vitesse d'un clou de cette roue, en chaque instant. L'article II.40 (la seconde loi de la nature) expose la pensée cartésienne sur ce point: la vitesse (ou plutôt la tendance à se mouvoir), elle aussi peut être jugée comme composée de deux (et donc de plusieurs) vitesses composantes (ou tendances à se mouvoir). En un sens, la situation s'en trouve simplifiée par rapport aux trajectoires composées et décomposées puisque les vitesses instantanées (ou encore, les tendances à se mouvoir) ne relèvent que de la ligne droite (voir ci-dessus). L'analyse de la tendance à se mouvoir ne fera apparaître que des lignes droites. C'est d'ailleurs sous cet aspect que la règle de composition peut apparaître, sous la forme dite «du parallélogramme ${ }^{41}$ ».

Descartes connaît bien ces questions. Il les connaît comme géomètre, mais aussi parce qu'elles furent décisives dans l'élaboration de la Diop$t_{\text {trique }}^{42}$. C'est là une raison supplémentaire d'examiner de près la determinatio dans les Principes, puisqu'elle constitue un des rares lieux proprement géométriques au sein de ce traité de philosophie naturelle. La Dioptrique, pas plus que la Géométrie, ne sont des parties de la physique cartésienne, mais voici qu'une notion venue de celles-là est nécessaire à celle-ci.

Descartes a obtenu la loi de la réfraction lumineuse par décomposition de l'inclinaison à se mouvoir de la lumière, pour laquelle il a expressément demandé à son lecteur d'accepter qu'elle suivait en cela les lois du mouvement.

car il est bien aisé de croire que l'action ou inclinaison à se mouvoir, que j'ai dit devoir être prise pour la lumière, doit suivre en ceci les mêmes lois que le mouvement. (Dioptrique, AT VI, p. 89)

40. Sur la notion cartésienne de vitesse, cf. Jullien, Charrak, etc.

41. Le jésuite Jan Ciermans donne, dans son cours publié en I640, Disciplinae mathematicae, la première expression de la loi du parallélogramme, sous forme trigonométrique. Dhombres, De Graves, p. I 8.

42. Il en fait un usage très performant dans les textes tardifs sur la chute des graves. 
De plus, il faut remarquer que la détermination à se mouvoir vers quelque côté peut, aussi bien que le mouvement et généralement que toute autre sorte de quantité, être divisée entre toutes les parties desquelles on peut imaginer qu'elle est composée; et qu'on peut aisément imaginer que celle de la balle qui se meut d'A vers B est composée de deux autres, dont l'une la fait descendre de la ligne AF vers la ligne CE, et l'autre en même temps la fait aller de la gauche $\mathrm{AC}$ vers la droite $\mathrm{FE}$, en sorte que ces deux, jointes ensembles, la conduisent jusqu'à B suivant la ligne droite AB (Dioptrique, AT VI, p. 94).

Une preuve du caractère controversé et innovant de la procédure cartésienne est fournie par les réactions extrêmement réservées de Fermat, qui n’a pas hésité à en contester la validité, sur le point précis de la composition des mouvements (ici des inclinations à se mouvoir). À propos de la décomposition selon les directions horizontale et normale de la lumière, sur laquelle s'appuie Descartes, Fermat objecte:

"Il n'a pris que celle qui lui peut servir pour sa conclusion; et partant, il a accommodé son médium à sa conclusion et nous en savons aussi peu qu'avant " (à Mersenne, septembre 1637).

La contestation de Fermat surprend d'ailleurs et montre assez qu'il n'est pas entré dans l'esprit de la règle de composition. En effet, dès lors que cette règle est adoptée, son objection tombe, car il est loisible de choisir toute décomposition en des parallélogrammes différents auxquels on ne demande que d'avoir une même diagonale.
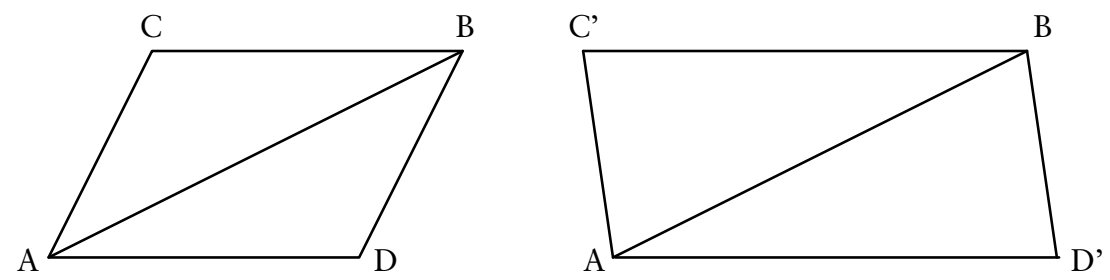

Soit $\mathrm{AB}$, l'inclinaison à se mouvoir résultante, elle est tout aussi bien le résultat de la combinaison de $\mathrm{AC}$ et $\mathrm{AD}$ que de $\mathrm{AC}$ ' et $\mathrm{AD}$ ', conformément à la figure ci-dessus.

Vingt ans plus tard, lors de la reprise de la polémique contre la démonstration cartésienne, le savant toulousain insiste:

L'usage de ces mouvements composés est une affaire très délicate qui ne doit être employée qu'avec de grandes précautions. Je la compare à ces médecines qui servent de poisons si elles ne sont pas bien et soigneusement préparées ${ }^{43}$.

D. Garber a donc raison de supposer que la notion de determinatio a pu naître dans les années I620, à propos de l'optique. Ce n'est pas une

43. À Cureau, I657, in Theories of Light, Sabra, CUP, I98 I, n. 21, p. I42. 
notion claire et distincte mais une notion émergente que propose Descartes; ainsi note Garber :

Il est juste de voir dans la notion cartésienne de détermination, une tentative sérieuse pour traiter quantitativement la notion de directivité du mouvement qui s'apparente à la notion moderne de vitesse (quantité vectorielle...) ou moment. Gabbey l'appelle «le mode directionnel de la force motrice ${ }^{44}$.

Le thème de la composition des mouvements, des vitesses et des forces occupe un grand nombre des savants du XVII ${ }^{\mathrm{e}}$ siècle; il n'est pas ici question d'en esquisser le traitement par les uns et les autres; seulement de comprendre sa présence dans les principes et un de ses principaux enjeux: la compréhension d'un objet nouveau qui associe une grandeur positive $s c a$ laire et des paramètres de direction.

La mention où l'on aperçoit avec le plus de netteté la chose en voie de constitution se rencontre dans la lettre du 3 décembre I653 au P. Bourdin. «[...] en parlant de la détermination vers la droite, écrit Descartes, j’entends toute la partie du mouvement qui est déterminée vers la droite ${ }^{45}$. La determinatio est ici traitée comme une des composantes du mouvement (ou plutôt de sa vitesse), c'est-à-dire comme une des pièces d'une chose qui plus tard sera nommée vecteur ${ }^{46}$.

Le commentaire des passages concernant la determinatio a largement consisté à examiner aussi précisément que possible les distinctions de notions présentes. Ce fut souvent en vain pour la simple raison que les notions évoquées ne sont justement pas distinctes. Il est question de mouvement, droit ou circulaire, de composante ou partie du mouvement; puis de tendance au mouvement, des causes qui concourent à déterminer son mouvement, de l'action pour se mouvoir, de détermination à se mouvoir d'un côté, d'inclination à se mouvoir, d'un effort pour aller vers divers côtés, de la force du mouvement, de la force de son agitation, et des diverses parties de cette force, de la force de la tension pour s'éloigner du centre ${ }^{47}$.

Le fait est que dans le mouvement d'un corps matériel plusieurs notions et concepts cohabitent que la mécanique moderne a peu à peu clarifiés: la trajectoire elle-même (le mouvement ou équation de mouvement), la vitesse qui accompagne nécessairement tout mouvement et l'accélération qui modifie l'état de mouvement quand elle n'est pas nulle. Or l'élaboration de ces notions n'est pas du tout achevée en ce moment cartésien; Descartes est l'un de ceux qui entrent dans ce labyrinthe.

44. Garber, La physique métaphysique de Descartes, p. 298.

45. AT III, 25I.

46. Il faut aller voir la lettre à Hobbes-Mersenne du 2I janvier I64I, p. 288, 1: 7-26. Sur l'actualité de la «composition des mouvements ", puis des vitesses, etc., et la réponse de Hobbes à Mersenne du 7 février I64I (AT III, 303). Un commentaire éclairant est donné par Damerov et al, «Exploring the Limits of Preclassical Mechanics », Springer-Verlag, I992, p. I I 8-I 20.

47. Voir notamment tout ce lexique dans le Monde VII et dans Pr. III, 57-59. 
Un seul exemple quant à l'état de la question à la même époque, celui $\mathrm{du}$ "traité de mécanique des poids soustenus par des puissances sur des plans inclinez à l'horizon» de Roberval ( ( 636). Les axiomes I et 2 du traité donnent les lois d'équilibre entre le poids et la réaction sur un plan incliné, le premier s'occupe des directions des forces, le second de leurs intensités. Les deux, ensemble, rendent compte de la situation par une notion très clairement vectorielle. Descartes connaissait ce traité dont il s'est d'ailleurs moquét8.

Pour un mouvement rectiligne (par exemple lors de la chute verticale des graves), ces diverses notions se présentent très simplifiées, car elles ont toutes une commune direction. Or, même dans ce cas, les difficultés sont sérieuses, comme le prouve la mise au point laborieuse du concept de vitesse instantanée. Dès lors que la trajectoire est courbe, les choses deviennent redoutables. La vitesse n'est pas de même direction que la trajectoire (ce que Descartes élucide avec sa tendance tangentielle) mais l'accélération tangentielle est nulle. L'accélération, qui n'est autre que la modification instantanée de la vitesse (d'un vecteur donc) correspond à une cause, à une force (ce que Descartes voit aussi puisqu'il écrit que «la pierre qui est dans la fronde fait tendre la corde d'autant plus fort qu'on la fait tourner plus vite» (Pr. III, 59). Cette force ne peut pas être dirigée comme la vitesse; Descartes voit encore qu'elle est déterminée par le centre de rotation (mention de la force que l'on sent en faisant tourner la fronde, qui est compensée par «l'effort que fait la pierre pour s'éloigner du centre $\mathrm{E} »(\operatorname{Pr}$. III, 57).

D'autres problèmes sont à considérer: comment se combinent (ou se composent) ces grandeurs (à supposer qu'il s'agisse bien de grandeurs)? Selon la règle du parallélogramme sans doute, mais une précision capitale doit être apportée. Seules se composent les grandeurs qui sont du même genre: on ne compose pas ensemble une accélération avec une vitesse, pas plus qu'une vitesse avec un élément de la trajectoire. Et cependant, chacune d'elles peut être décomposée selon des directions choisies. On pourra lire la grande lettre à Huygens de février I 643 sur la trajectoire du jet d'eau pour se convaincre de la précision avec laquelle Descartes —comme Galilée, ou sous son influence - démontre la trajectoire parabolique des corps soumis à la gravité et à une vitesse initiale horizontale (ou oblique); il le fait en composant les mouvements, ou plutôt les déterminations à se mouvoir ${ }^{49}$. Regardons les choses de plus près: que doit-on composer pour démontrer la trajectoire parabolique? Elle dérive de la coexistence d'un mouvement vertical uniformément accéléré et d'un mouvement horizontal uniforme inertiel (pour prendre une situation simple); on ne compose pas cependant une accélération (ou une force) verticale avec une vitesse horizontale, ce qui

48. On pourrait longuement mentionner les Thèses de Grégoire de Saint Vincent, soutenues à Louvain en I 624, où la chose vectorielle est si prégnante (voir Dhombres-Radelet 2009, P 57-58).

49. I8-I9 février I643, A.T. III, p. 6I9-63 I. 
n'aurait pas de sens, mais bien deux vitesses dont l'une est constante (l'inertielle horizontale) et l'autre est modifiée à chaque instant conformément à la loi de la chute des corps. Ce sont de telles distinctions qui sont à l'œuvre sous le terme confus de determinatio.

En conclusion de leur chapitre Conservation and contrariety, Damerov et al. estiment que:

Descartes is probably the only person who ever used his concept of determination productively in physic. [...] It proved almost impossible to apply the concept of determination to cases which Descartes had not already explained. It was not an everyday tool that could be used by any competent scientist; it was an idiosyncratic instrument that could be employed only by his inventor ${ }^{50}$.

Les articles III. 58 et III.59 où le modèle de la fronde est réactivé, rendent manifestes ces difficultés. Pour mieux faire comprendre la chose à son lecteur, Descartes met en scène une fourmi qui se déplace sur une règle elle-même en rotation uniforme. Cette fourmi doit avoir un comportement bien étrange pour demeurer sur la droite notée ACG: elle doit parcourir des distances, à partir de $\mathrm{A}$, qui sont données en fonction de $t(t$ est le temps qui mesure la rotation); cette fonction doit être $d(t)=(1-\cos t) /$ cost dont on se demande bien quel phénomène pourrait la produire. Le moins qu'on puisse dire est que le commentaire de ce mouvement est assez désinvolte à l'article III.59 qui pourtant est une explication de ce déplacement:

Je ne doute point que le mouvement de cette fourmi ne doive être très lent au commencement, et que son effort ne saurait sembler bien grand, si on le rapporte seulement à cette première motion; mais aussi on ne peut pas dire qu'il soit tout à fait nul, et d'autant qu'il augmente à mesure qu'il produit son effet, la vitesse qu'il cause devient, en peu de temps, assez grande.

Descartes a recours à « une autre comparaison. "Que la petite boule A soit mise dans le tuyau EY, et voyons ce qui en arrivera" ".

Lorsqu'il écrit «la pierre qui est dans une fronde fait tendre la corde d'autant plus fort qu'on la fait tourner plus vite", il ne peut ignorer qu'il écrit grossièrement, car «d'autant plus fort» exprime, au propre, une proportionnalité; ici, il n'y a pas de proportionnalité. Si $t$ est multiplié par $k$, l'accélération de la fourmi ou de la bille n'est pas multipliée par $k$, mais par un facteur beaucoup plus compliqué et "non algébrique». La phrase finale, "nous pouvons connaître par cette tension (qui est la réaction centrifuge à l'action centripète) quelle est la quantité de cet effort » est vraiment intéressante, elle suppose que Descartes peut calculer la modification de vitesse (c'est-à-dire la cause du changement de mouvement ou, en terme moderne, l'accélération). Or sa Géométrie ne prépare pas ce type de calcul, et quand bien même lui-même parvient à ce genre de résultats, il ne les qualifie pas

50. Damerov et al, «Exploring the Limits of Preclassical Mechanics », Springer-Verlag, I992, p. I24. 
de connaissance certaine (sinon relevant de la certitude morale). En outre, le «mouvement» de A vers G, qui représenterait la composante tangentielle du mouvement n'est pas inertiel dans cette situation: on a en effet «AM = tg.t ».

On comprend rétrospectivement la luxuriance du vocabulaire, et il est vain de chercher à y reconnaître des concepts stables. Les tendances, déterminations, forces d'agitation, etc., cartésiennes ne sont que des pistes, des brouillons, des anticipations des concepts d'une mécanique en gestation. On perdrait son temps à chercher à en stabiliser le contenu conceptuel, ce qui n'est pas la même chose que de chercher à comprendre et à reconstituer le cheminement difficile qui mène aux solutions classiques.

Il est déjà remarquable que Descartes ait pu dégager la notion de mouvement inertiel selon la tangente, celle de force centrifuge, et qu'il ait proposé certaines considérations décisives pour concevoir ce que seront les grandeurs vectorielles (determinatio, intensité) ${ }^{51}$.

P. Damerov, G. Freudenthal, P. McLaughlin et J. Renn ont parfaitement raison d'écrire:

The difference in status between scalar and vector conservation laws in classical mechanics and the philosophical relevance of this difference has, to our knowledge, never been seriously studied $[\ldots]$, the $\mathrm{I}^{\text {th }}$ century, the system was defined by the real and positive (i.e., scalar) magnitudes not by those that are merely modal (e.g., directional), even though the latter are necessary for calculating the trajectories of colliding particles ${ }^{52}$.

Un aspect essentiel de la determinatio est qu'elle se présente comme convenant aussi bien à une vitesse (donc à un mouvement actualisé) qu'à une accélération ou une modification de vitesse (donc à un mouvement en voie d'actualisation, à une tendance si on veut). Elle relève du conatus comme de l'impetus. Elle est comme une forme valable pour des grandeurs physiques de nature différente; tel sera bien entendu l'outil vectoriel. Il faut toutefois reconnaître que le lexique cartésien (à l'article II. 57 par exemple) n'aide pas à voir clairement cette propriété essentielle puisque la «force de son mouvement (celui de la pierre) » concerne d'abord la tangente (c'est une vitesse ponctuelle), puis, «elle (la pierre) fait effort pour s'éloigner du centre» (c'est la réaction à l'accélération centrifuge).

Des commentateurs ${ }^{53}$ ont insisté sur ce qu'il est convenu d'appeler le «Principe de Clerselier», ce qui permet aux philosophes de retrouver un sol

51. À vouloir reconnaître des concepts stables, on risque l'erreur. Il n'est pas juste de dire que «en chaque point du cercle [...] s'exercent deux forces perpendiculaires [...] l'une suivant la tangente [...] l'autre suivant la normale» (bc, p. Iо4 dans le commentaire de III. 57). Il n'y a pas de force suivant la tangente, il y a une vitesse instantanée. On a là un exemple de composition d'une vitesse et d'une accélération (ou force).

52. Damerov et al., "Exploring the Limits of Preclassical Mechanics », Springer-Verlag, I992, p. 76.

53. Notamment D. Garber, de Buzon et Carraud. 
plus assuré puisqu'il est question des modes du mouvement et apparemment pas d'une confuse notion de physique en voie de constitution. Est-ce si sûr?

Lorsque deux corps se rencontrent, qui ont entre eux des modes incompatibles (les modes du mouvement étant la vitesse scalaire et la determinatio, $c f$. art. 4I et 44), il se doit véritablement faire quelques changements en ces modes, pour les rendre compatibles, mais ce changement est toujours le moindre qui puisse être, c'est-àdire que, si certaine quantité de ces modes étant changée ils peuvent devenir compatibles, il ne s'en changera point une plus grande quantité $e^{54}$. (p. 185)

Garber le nomme Principe du moindre changement modal ${ }^{55}$ et explicite son fonctionnement en détail. Ce principe est censé fournir des conditions de résolution de certains cas de choc mais, évidemment, il ne nous enseigne pas comment quantifier la determinatio: deux determinationes considérées dans ces divers cas ne peuvent être qu'identiques ou opposées et non pas mesurées par une échelle numérique. La chose est ici presque fonctionnelle car on est réduit à deux directions opposées, une sorte de situation binaire; mais que devient le "principe de Clerselier» dans le cas des chocs obliques?

L'histoire de la physique a enregistré un principe très général en vertu duquel une grandeur n'entre en physique que quand elle devient objet possible d'opérations. Il est certain que si la determinatio est seulement la direction, elle n'est pas une grandeur physique, alors que la force scalaire l'est immédiatement. C'est pourquoi il paraît difficile, impossible même, de voir dans la determinatio seulement la direction ${ }^{56}$, dès lors que cette notion est candidate au statut de grandeur physique. Irrésistiblement, il y a dans la determinatio l'idée de la direction et aussi l'idée de quantité. Or une direction ne semble pas pouvoir être quantifiée. Quoique! Dans une lettre à Arnauld, Leibniz exprime la determinatio comme la quantité de direction; allant plus loin, il suggère que les déterminations ou les directions (synonymes?) peuvent se calculer, s'additionner (on peut considérer la "somme des directions ou déterminations), etc. Il mentionne encore "la somme des quantités de toutes les directions". Il apparaît donc que Leibniz lit chez Descartes, avec determinatio, une grandeur qui se mesure, pas seulement une direction ${ }^{57}$. notait $^{58}$ :

Pierre Costabel avait nettement observé l'enjeu de ces passages lorsqu'il

N'est-il pas entendu que c'est à ce grand auteur que l'on doit d'avoir avancé d'un pas pour la dotation vectorielle indispensable au traitement mathématique

54. Lettre à Clerselier du I7 février I645, AT IV I 85 , Alquié, III, 557.

55. D. Garber, La physique métaphysique de Descartes, p. 372.

56. Les notions d'estime, de repérage ou de mesure sont attachées à ce mot (voir son sens juridique où il est même l'acte de fixation des bornes).

57. "Lettre de Leibniz à Arnaud du 30 avril I687", Discours de métaphysique et correspondance avec Arnaud, Vrin, I970, p. I62.

58. Démarches originales de Descartes savant, «Essai critique... », p. I4I-I42. 
des questions de mécanique, et cela par la reconnaissance distincte de la direction adjointe au scalaire de la vitesse?

Costabel tentait - il était là moins convaincant sans doute - une manière de définition ou de caractérisation de la determinatio cartésienne qui serait "une condition géométrique de direction qui s'impose au mouvement, qu'il soit en acte ou en puissance ", il ajoutait que c'est comme une condition d'actualisation du mouvement en puissance (p. I45).

Encore une fois, Damarov et al. voient juste lorsqu'ils notent

Determination, as he [Descartes] elsewhere makes clear (cf. Dioptrics), has a direction and a magnitude both of which are conserved only in absence of interactions [...] Thus, it would seem that the use of the parallelogram rule $[\ldots]$ is one of the essential elements of Descartes' system $^{59}$.

On trouvera un écho parfaitement net de cette interprétation de la determinatio cartésienne dans l'usage qu'en fait Isaac Newton ${ }^{60}$. Celui-ci présente la méthode de Roberval pour les tangentes qui consiste en une remarquable utilisation de la composition des vitesses ponctuelles, valable pour certaines catégories de courbes ${ }^{61}$ :

In the description of any Mechanical line what ever there may be found two such motions which compound or make up the motion of the point describing it and by those two motions may the motion of the point bee found [...] whose determination is in a tangent $[\ldots]$ to the crooked line.

Comme le Remarque Panza, "Whiteside suggère que Newton emprunte sa terminologie aux Principia de Descartes", et il ajoute qu'en effet, "la notion cartésienne de détermination d'un mouvement ne se réduit nullement à celle de direction de ce mouvement, cela incluant aussi la composante scalaire de la vitesse ${ }^{62}$ ». Plus loin, Panza juge ainsi que

Newton semble s'appuyer sur une conception [...] du mouvement, en insistant sur la double nature des propriétés qui caractérisent un mouvement particulier: d'un côté une qualité intensive, ce qui revient dans notre langage à une grandeur scalaire; de l'autre un positionnement dans l'espace, ce qui est pour nous une direction. Ce qui est nouveau chez Newton, c'est l'effort de condenser en une seule notion ces deux caractéristiques ${ }^{63}$.

59. Damerov and al., id. p. 77. On lira avec profit les pages I03-I06 de cet ouvrage, où l'analyse de la determinatio cartésienne montre nettement les liaisons qu'elle entretient avec l'élaboration future de la grandeur vectorielle.

60. Voir en particulier sa note du 30 octobre I665. Elle est analysée avec soin par Marco Panza, Newton et les origines de l'analyse: 1664-1666, Paris, Albert Blanchard, 2005, p. 360 sq. 61. Donner les références du dossier.

62. Panza, note 54, p. 360.

63. Panza, p. 36I. 


\section{Les dimensions du système stellaire}

La troisième partie des Principes s'ouvre sur un argument métaphysique selon lequel nous ne pouvons surestimer la grandeur, la beauté et la perfection de la création, et l'erreur viendrait d'y supposer des bornes ou des limites $^{64}$. Il faut donc s'attendre à un monde immense.

La longue série d'articles (de III.5 à III.47) au cours desquels Descartes va discuter des hypothèses astronomiques et exposer la sienne est caractérisée par la faible technicité de ces articles. Ils sont à la fois extrêmement précis et conformes aux connaissances les plus récentes ${ }^{65}$, mais dépourvus de justifications qui relèveraient de l'astronomie professionnelle ou géométrique: pas de modèles, de calculs, de mesures (ou juste celles qui sont nécessaires à l'admission de son système). Il faut cependant savoir que Descartes connaît fort bien cette astronomie technique et professionnelle, de terrain et de calcul (il l'a pratiquée avec Lansbergen notamment).

C'est comme si, reprenant l'antique controverse qui a pu être présentée comme opposant un certain platonisme à Aristote sur le programme de l'astronome, il avait choisi le programme aristotélicien ${ }^{66}$ : dire les Principes et ne pas se contenter des modèles et systèmes géométriques qui "sauvent les phénomènes". Il expose le pourquoi, pas le comment, du mouvement des astres (comme Aristote dans le Traité du ciel). Il ne s'agit pas, ici, d'exposer le contenu positif et les caractéristiques épistémologiques de l'astronomie et de la cosmologie cartésienne; il suffira de dire que Descartes forge un concept de Monde général et cohérent. Cette partie est si riche, si audacieuse, si prémonitoire que les jugements qui font de Descartes un astronome ou cosmologue timoré et pusillanime sont parfaitement insoutenables.

Très tôt, Descartes s'est préoccupé de la possibilité des dimensions indéfinies de l'Univers. Dans la lettre à Mersenne du I 8 décembre I 629 il veut savoir "s'il n'y a rien de déterminé en la religion, touchant l'étendue des choses créées...» (AT. I, 86).

La question de la taille de notre système stellaire ${ }^{67}$ occupe décidément une place privilégiée. On verra qu'il est crucial de faire admettre un système du monde immense qui, seul, peut justifier l'héliocentrisme. Les articles 5, 6, 7 et 8 s'occupent des dimensions possibles ou établies des objets célestes et de leurs éloignements que nous "connaîtrons par des raisonnements qui sont infaillibles » : en 5 il s'agit de la Terre, de la Lune et du Soleil, en 6, des

64. Voir $\operatorname{Pr}$ III. I.

65. Voir Lerner, Le monde des sphères, vol. 2, Paris, Les Belles-Lettres, I997 et Bucciantini, Képler, Galilée, Paris, Les Belles-Lettres, 2009.

66. On pense ici aux thèses de Pierre Duhem telles qu'elles sont soutenues dans Sauvez les phénomènes.

67. J'appelle système stellaire l'ensemble formé par notre soleil, ses planètes et les étoiles visibles, dites fixes. 
autres planètes et en 7 , des fixes ${ }^{68}$. Le cartouche de l'article 7 annonce "qu'on peut supposer les étoiles fixes autant éloignées qu'on veut »; elle est de grande importance. Ces quatre articles et l'article I 3 assimilant le Soleil à une étoile préparent les articles 20 et 23 qui reviendront sur le même sujet, et surtout les articles 40 et $4 \mathrm{I}$ où l'enjeu fondamental de la question est explicité.

De quoi s'agit-il ? De la très ancienne question de la parallaxe stellaire dont je donne ici un très bref aperçu. Soit conçu un cosmos dont les limites sont constituées par une "sphère des fixes". Sa dimension sera donnée par le demi-diamètre cosmique, c'est-à-dire la distance du centre du cosmos aux étoiles. Il est connu depuis l'antiquité que le choix d'une hypothèse héliocentrique agrandit nécessairement le cosmos, et ce dans des proportions énormes.

La raison en est fort simple et se comprend par un argument géométrique élémentaire, nommé parallaxe stellaire.

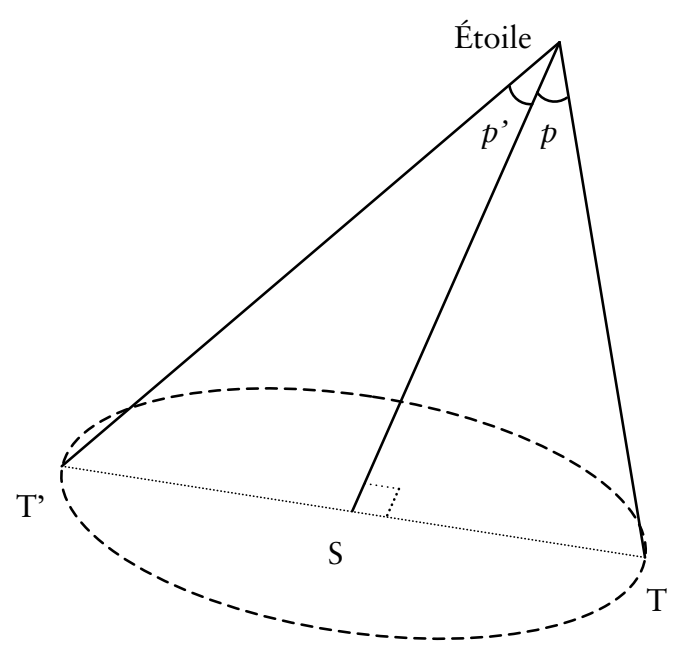

La Terre $T$ est en T' six mois plus tard. Les positions T et T' sont choisies de façon à ce que les angles TSE et T'SE soient droits, ce qui arrive une fois l'an. La parallaxe est la valeur de l'angle $p$ (ou $p$ ' six mois plus tard). C'est «l'angle sous lequel on mesure la distance TerreSoleil, à partir de l'étoile E. Il est clair que l'angle $\mathrm{p}$ doit varier, étant donné l'immense «circuit» accompli par la Terre autour du soleil.

Or nulle variation de la parallaxe ne put être mesurée, ni par Hipparque, Aristarque ou Ptolémée dans l'antiquité, ni par

Copernic, Tycho Brahe, Képler ou Galilée à l'époque moderne. Ce qui aurait constitué un argument irrésistible en faveur de l'héliocentrisme se retourne en une objection très efficace contre ce système.

68. Une remarque: les méthodes géométriques pour évaluer la distance Terre-Lune, puis Terre-Soleil sont examinées depuis les anciens (Hipparque, Ptolémée, etc.). La méthode TerreLune est fiable et les 30 diamètres terrestres sont conformes.

En revanche, le raisonnement géométrique pour Terre-Soleil (appelée UA), s'il est fondé sur une "bonne idée » est tout-à-fait impraticable (la raison en est que toute imprécision sur la mesure de l'angle "Lune-Terre-Soleil» a en distance des conséquences gigantesques). L'UA de Copernic vaut I I 42 rt, de Ptolémée I 200 rt, de Descartes ou Kepler I400 rt. Elle sera reconsidérée au XVIII ${ }^{e}$ par la méthode des parallaxes solaires et des passages de Mercure et Vénus, et évaluée à 20 fois plus grande. Les valeurs sont toutes relatives. Un système vrai «à une homothétie près ". 
Le problème est bien connu, et Archimède en avait fait un point central de l'organisation de son traité L'arénaire. La seule solution pour justifier le mouvement de la Terre autour du Soleil doit soutenir que la variation parallactique existe bien mais qu'elle est indétectable. Si elle est indétectable, c'est parce que les étoiles sont beaucoup plus éloignées de la Terre (et du Soleil) que dans le système où elle ne se meut pas. Ainsi, le cosmos d'Aristarque de Samos était-il immense en comparaison des dimensions de celui d'Aristote ou de celui de Ptolémée.

Lorsque Descartes intervient, les données sont nettes: la distance Terre-étoiles acceptée traditionnellement par les géocentristes est de $0,13.109 \mathrm{~km}$ (on modernise les unités et les notations), et la distance soutenue par Kepler est quelque part autour de $250.109 \mathrm{~km}$ (elle changera, mais en devenant plutôt plus grande). Le système de Tycho Brahé échappe à l'objection parallactique, et l'astronome danois rectifie les mesures de distances pour donner un cosmos d'un diamètre de 0,09.I09 km.

Comme le montre cette troisième partie des Principes, le système $d u$ monde qui doit être le plus sévèrement combattu par Descartes est celui de Tycho ${ }^{69}$. Vraisemblablement, l'explication est à chercher du côté de la Compagnie de Jésus dont plusieurs figures éminentes penchaient en faveur du système semi-héliocentrique, qu'il fût de Tycho ou encore une de ses variantes comme par exemple celle du Père Riccioli ${ }^{70}$. Or Tycho Brahe s'était montré particulièrement sensible à l'argument parallactique qui semble avoir été pour beaucoup dans son choix de combattre les hypothèses héliocentriques $^{71}$. Non seulement, la taille formidable du cosmos keplerien lui semble "absurde», mais encore ses propres calculs et observations le conduisent-ils à réduire celui de Ptolémée jusqu'à $0,09.109 \mathrm{~km}$. Une conséquence saisissante de cette controverse résulte de la simple remarque suivante: si l'on accepte de multiplier les distances par environ 2000 (de Ptolémée à Kepler), on multiplie alors le volume du cosmos, ou le système du monde par 8 milliards, ce qui ne peut laisser d'impressionner même les imaginations les plus maîtrisées. Il va de soi que de redoutables questions gisent là derrière: par exemple ces deux-ci: les dimensions immenses suggérées sonnent le glas des sphères contiguës: le calcul de Tycho, dérivant du système de Copernic, donne entre la sphère de Saturne et les fixes un «vide» de quelque 8 millions

69. Contrairement à Galilée, du moins si l'on se conforme au titre de son livre sur Les deux principaux systèmes... au nombre desquels ne figure pas celui de Tycho. La réalité est sans doute plus complexe, et le poids des arguments et des activités tychoniennes sur Galilée furent considérables, comme le montre bien Bucciantini dans son livre Kepler et Galilée.

70. Jean Dhombres et Patricia Radelet de Graves ont montré que des milieux savants jésuites, autour de Grégoire de Saint Vincent, étaient nettement convaincus de la validité du système héliocentrique. Voir Une mécanique donnée à voir, Brepols, 2009, p. Io s sq.

71. Sur tout cela il y a beaucoup de bonne littérature; on mentionnera ici seulement $L e$ monde des sphères II, M. Lerner, Paris, Belles-Lettres, et Kepler et Galilée, M. Bucciantini, trad. française, Belles-lettres, 2009. 
de rayons terrestres. La discussion précédente est évidemment simplifiée, voire faussée si l'on songe que la controverse n'est pas: sphère des fixes proches contre sphère des fixes lointaines parce que souvent, pour certains protagonistes (c'est au premier chef et admirablement le cas de Descartes), la sphère des fixes elle-même cesse d'être un concept valide de la cosmologie. C'est d'ailleurs sur tous ces points qu'intervient Descartes au cours de la troisième partie des Principes. Au total, la question des dimensions du système est un point d'appui (le point d'appui le plus sensible peut-être) qui permet de développer l'ensemble de l'hypothèse cartésienne; c'est ce que dira explicitement l'article 40.

Si l'on est attentif à l'état des controverses astronomiques et cosmologiques de la période, et si, en outre, on examine de près les recherches cartésiennes sur le problème, on ne peut être surpris de l'importance donnée à la parallaxe dans le traité (quoique le mot ne soit pas prononcé). Descartes a pris cette question très au sérieux, $\mathrm{y}$ compris du point de vue technique, et il rend compte d'une observation faite le 20 novembre $\mathrm{I} 642$ par laquelle il a cherché si on ne pouvait pas observer le mouvement terrestre dans le fond du ciel; c'est bien la variation de parallaxe qu'il essaie de débusquer (comme Kepler, comme Galilée, comme Newton plus tard... comme tous les astronomes jusqu'à Bessel en I 838) où il recherche explicitement une mesure de la parallaxis: "ad observandum an parallaxis aliqua [...] $]^{72}$ ». L'article III.4O "renonce " à la mesure de parallaxe, sans renoncer au système en justifiant à nouveau le caractère indétectable de cette variation angulaire par les dimensions aussi grandes qu'il le faut du système du monde. Cet article confère bel et bien un rôle stratégique à l'objection de la parallaxe:

On peut ici proposer une difficulté contre mon hypothèse, à savoir, etc. [...] et néanmoins on n'en a rien su encore découvrir par les observations qu'on a faites. Mais il est aisé de répondre que la grande distance qui est entre la Terre et les étoiles en est cause: car je la suppose si immense, que tout le cercle que la Terre décrit autour du Soleil, à comparaison d'elle, ne doit être compté que pour un point. Ce qui semblera peut-être incroyable à ceux qui n'ont pas accoutumé leur esprit à considérer les merveilles de Dieu, et qui pensent que la Terre est la partie principale de l'univers, parce qu'elle est la demeure de l'homme, en faveur duquel ils se persuadent sans raison que toutes choses ont été faites; mais je suis assuré que les astronomes, qui savent déjà que la Terre, comparée au ciel, ne tient lieu que d'un point, ne le trouveront pas si étrange (Pr. III. 40).

Quelques remarques complémentaires, très succinctes mais aussi très importantes, méritent d'être avancées. Résumons-les ainsi: Descartes ne propose pas de trancher entre trois hypothèses concurrentes, de Ptolémée,

72. Excerpta, ATXI, 650. Ce passage est expliqué et commenté par Gaston Floquet en AT XI, p. 696-697. Les dimensions énormes sont aussi nécessaires pour rendre compte du mouvement des comètes. Tycho n'avait pas osé les faire dépasser Saturne (cf. art. 4I). 
de Tycho et de Copernic. Son objet est d'établir sa propre hypothèse; elle est, en un sens général, copernicienne puisqu'héliocentrique, mais elle est bien autre chose. Descartes apporte des réponses globales qui constituent, ensemble, un nouveau concept de monde; telle est la fonction des articles 20

à 29:

Art 20. Les étoiles sont très éloignées, au point que leurs distances à notre système sont au-delà des analogies d'éloignement entre choses terrestres. L'article 20 est clairement rédigé contre Tycho qui jugeait absurdes ces énormes distances. L'acceptation de celles-ci sera «utile à notre dessein » souligne Descartes, et c'est là un point important puisqu'il indique que le dessein en question consiste notamment à briser la voûte étoilée; cet article est aussi contre Galilée et sa conception prudente devant l'infinité de l'Univers.

Articles 21 à 23. Le Soleil et les étoiles ont même nature. Le Soleil fait partie, avec les étoiles, d'un système stable au sein duquel il n'y a pas de mouvement relatif. Cela conduit à la négation de la sphère des fixes, ce qui constitue une prise de position nette dans un débat de grande actualité ${ }^{73}$.

Article 24-25. Les cieux sont liquides. "En troisième lieu, pensons que la matière du ciel est liquide, aussi bien que celle qui compose le Soleil et les étoiles fixes. C'est une opinion qui est maintenant communément reçue des astronomes $[\ldots]$ ». Descartes note que, pour les astronomes, il s'agit d'une proposition instrumentaliste et non pas de principe (parce qu'ils voient qu'il

73. Copernic opte pour la finitude, quoiqu'il semble hésiter, et une de ses formules mérite d'être citée: la concavité de la sphère du monde relève de l'astronomie, sa convexité, de la théologie. Copernic examine avec beaucoup de soin la thèse de l'infinité et semble finir par la délaisser. Il «ne paraît pas rejeter comme absurde l'idée que le ciel «fini par sa concavité intérieure » puisse être infini à l'extérieur [...] Il s'interdit de trancher, laissant cette délicate question en suspens, et son éventuelle solution aux philosophes de la nature» (Lerner, p. 99). Galilée réussit l'exploit de ne pas se prononcer nettement sur la finitude du monde. Il reste prudent sur la question de la finitude, mais, tout en soutenant qu'elle ne peut pas être tranchée rationnellement, il incline plutôt vers l'infinité: "Il y a une raison qui m'est bien particulière d'incliner plutôt en faveur de l'infini que du limité, dès là que je ne connais ni ne peux imaginer l'univers ni limité, ni illimité et infini: puisque l'infini, de par sa nature propre, ne peut pas être compris par notre intellect limité [...] Mais c'est là une des questions qui se trouvent être inaccessibles aux raisonnements humains [...] " (lettre à Liceti en 1640).

«Ne savez-vous pas qu'il est encore indécidé (et je crois que cela le restera toujours pour les sciences accessibles à l'homme) si l'univers est fini ou infini ? [...]».

Kepler est «résolument finitiste, et sa position est renforcée après la publication du Sidereus nuncius de Galilée (en I 609). Pour Kepler, les étoiles fixes forment un firmament assez traditionnel.

Comme le dit Lerner, la question du monde comme «immense et semblable à l'infini » (Pline) est indécidable du point de vue de l'observation astronomique, et les partisans comme les adversaires du système de Copernic se partageront en deux camps. Dans le premier camp, on trouvera des coperniciens et des anticoperniciens pour défendre le maintien d'une sphère des fixes de structure classique, quelles que soient ses dimensions. Dans le deuxième camp on trouvera des adeptes de l'héliocentrisme, mais aussi des auteurs hostiles à la nouvelle cosmologie pour considérer que [...] la sphère des fixes cessait d'être évidente (d'après Lerner, p. I০০-IOI). 
est presque impossible sans cela de bien expliquer les phénomènes). La question des cieux fluides (ou, pour D., des tourbillons) va changer de statut au cours de Pr. III. Elle est d'abord une hypothèse très performante ("pensons que la matière du ciel est liquide », puis prend statut d'une thèse nécessaire. Il va de soi que cela est associé aux articles de la fin de $\mathrm{Pr}$. $\mathrm{II}^{74}$.

Articles 26-29. La Terre et les planètes sont transportées. L'important n'est pas tant que la «Terre se repose en son ciel », mais bien sûr "qu'elle ne laisse pas d'être transportée par lui " et "qu'il en est de même de toutes les planètes" $(27)^{75}$.

Simone Martinet ${ }^{76}$ a excellemment commenté cette thèse cartésienne:

On sait en effet qu'à la suite de Tycho les astronomes avaient brisé la solidité des orbes coperniciens, pour pouvoir notamment expliquer les trajectoires des comètes. Mais alors que la liquidité des cieux n'avait été admise par Tycho que comme une condition sans laquelle on ne pouvait comprendre les phénomènes, elle devient pour Descartes le postulat grâce auquel on peut expliquer le transport des planètes [...] Alors que le système tourbillonnaire [...] devient une conséquence des principes des choses matérielles qui avaient été exposés au Livre II. [...] On voit donc que Descartes ne se borne pas à ratifier l'hypothèse de Copernic, mais l'intègre dans un système plus vaste [...] avant même de conférer à l'hypothèse ainsi modifiée l'évidence de la science parfaite. [...] Leibniz disait que "son système du monde, quel qu'imaginaire qu'il soit, est pourtant si beau qu'il peut servir de modèle à ceux qui chercheront les causes véritables" (Gerhardt, IV, p. 308) et d'Alembert écrivait, dans le Discours préliminaire de l'Encyclopédie: "Si l'on juge sans partialité ces tourbillons devenus aujourd'hui presque ridicules, on conviendra, j'ose le dire, qu'on ne pouvait alors imaginer rien de mieux.»

On n'a pas ici discuté des problèmes proprement épistémologiques posés par cette séquence longue et complexe, concernant le statut des hypothèses, l'intégration des résultats techniques de l'astronomie contemporaine, la quasi absence d'argumentation géométrique et mathématique dont Descartes utilise pourtant les résultats. On a voulu - comme pour les deux thèmes précédents, la relativité du mouvement et la determinatio - montrer à quel point les controverses scientifiques précises, l'élaboration en cours des concepts scientifiques irriguaient le traité cartésien et montrer aussi que, dans une certaine mesure, celui-ci ne se laissait vraiment comprendre qu'à la lumière d'une prise en considération aussi précise que possible de ces efforts.

74. Voir III.46 et IV.206 qui soulignent que la thèse des cieux fluides concentre "tout ce que Descartes a supposé» (IV, 206).

75. Cette thèse cartésienne fait écrire à Lerner (Le monde des sphères, II, p. I79): «En sorte que les "cieux liquides» emporteraient la Terre considérée comme en repos [...] exactement comme auraient pu le faire les orbes «solides" traditionnels.» Le péripatéticien Liceti soutenait quelques années avant Descartes que les planètes seraient entraînées par des orbes "fluides" comme le sont l'eau et l'air (cf. note 232, p. 303 ).

76. AAA Copernic, Paris, Blanchard, I975, p. 238-239. 